\title{
Emittance growth in the NLCTA first chicane
}

\author{
Yipeng Sun* and Chris Adolphsen \\ SLAC, Stanford, CA 94025, USA
}

\begin{abstract}
In this paper, the emittance growth in the NLCTA (Next Linear Collider Test Accelerator) first chicane region is evaluated by simulation studies. It is demonstrated that the higher order fields of the chicane dipole magnet and the dipole corrector magnet (which is attached on the quadrupoles) are the main contributions for the emittance growth, especially for the case with a large initial emittance $\left(\gamma \epsilon_{0}=5 \mu m\right.$ for instance). These simulation results agree with the experimental observations.
\end{abstract}

\section{Overview}

The NLCTA is a test accelerator at SLAC, which is roughly 42 meter long and composed of X-band accelerator structures [1]. The aim is to develop and demonstrate the $\mathrm{X}$-band $\mathrm{rf}$ acceleration technologies for the next generation linear collider, with an accelerating gradient of $50 \mathrm{MV} / \mathrm{m}$ to $100 \mathrm{MV} / \mathrm{m}$.

The main components of NLCTA are, consecutively, a thermionic-cathode electron gun (a beam energy of $5 \mathrm{MeV}$ ), an X-band buncher $(60 \mathrm{MeV})$, a magnetic chicane, six 1.8-meter-long sections of 11.4-GHz acceleration structure, and a magnetic spectrometer [1]. A sketch of the accelerator from the electron gun to the exit of the first chicane is shown in Figure 1 below.

In the routine operation of NLCTA, it is observed that the transverse beam emittance is increased from $5 \mu m$ (exit of RF gun) to around $10 \mu m$ (exit of chicane 1), with all the 12 quadrupoles between the dipoles turned off. The emittance growth can be as large as 5 to 10 times, with the quadrupoles between the dipoles turned on.

In the following sections, the possible sources of emittance growth is evaluated by multi-partilce tracking simulations. A new code is used for the special treatment of the higher-order fields of the steering dipole correctors [2]. The

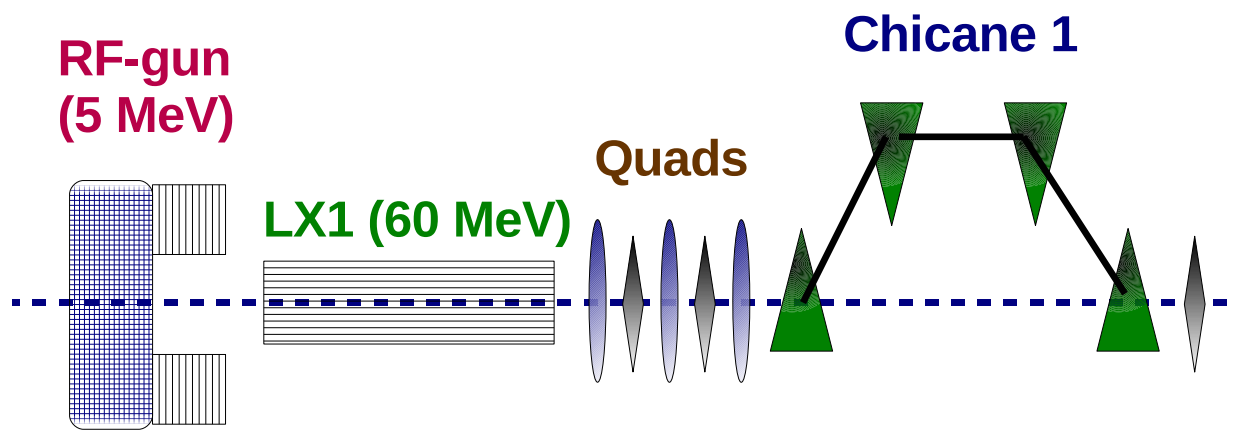

Figure 1: Sketch of the NLCTA accelerator, from the thermal RF gun to the exit of first chicane.

*Electronic address: yisun@slac.stanford.edu 


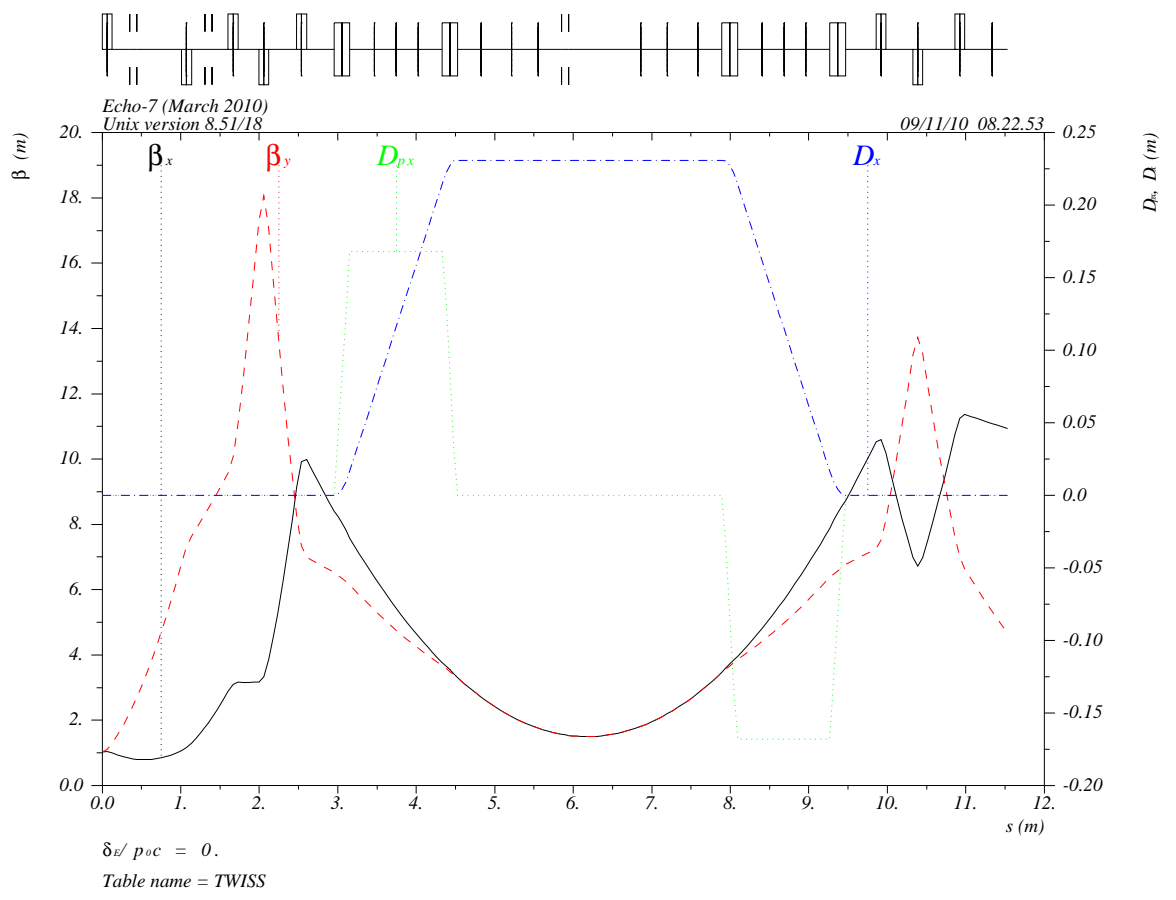

Figure 2: NLCTA optics of the normal operating case [3].

simulation results with two different steering methods (one-to-one steering and one-to-few steering) are compared. It is observed that with one-to-few steering technique applied, the emittance growth is much smaller.

\section{Optics}

There are twelve quadrupoles between the four dipole magnets in the first chicane, which are designed to adjust the pathlength-energy dependence in the first place $\left(R_{56}\right.$ in the first order transfer matrix). However, during the operation of NLCTA, it is observed that the beam emittance will increase dramatically if one applies the optics which makes use of these quadrupoles. One possible reason for the huge emittance growth could be misalignment of these quadrupoles plus that they are located in a high dispersion region (with a maximum dispersion of $0.23 m$ ). At the same time, higher order magnetic fields and steering effect (one-to-one) are also possible sources.

Thus, in the normal running mode of NLCTA, these quadrupoles are all turned off, in order to better preserve the emittance. In Figure 2, the first order NLCTA optics is shown for the region between the X-band buncher exit and the fourth quadrupole after the first chicane exit [3]. There are a pair of steering dipole magnets and a Beam Position Monitor (BPM) attached on each quadrupole magnet.

The electron beam energy is $60 \mathrm{MeV}$ in this 12 -meter-long region. There are five quadrupoles before the chicane and four quadrupoles after the chicane. Each dipole magnet has a length of $0.2 \mathrm{~m}$ and provides a bending angle of 9.6 degree. The quadrupole length is $0.126 m$ and they have an average normalized strength of $14\left(K=\frac{\partial B / \partial x}{B \cdot \rho}\right)$. Assuming a half vertical gap of $1.5 \mathrm{~cm}$ and taking the beam energy $60 \mathrm{MeV}$ into account $(B \cdot \rho=2 k G-m)$, the average quadrupole pole-tip field is roughly $0.5 k G$. 


\section{Emittance growth simulations}

\subsection{Code and simulation setup}

The simulation study is performed in a new Fortran90 code, which is optimized to implement different kind of steering algorithms in a linear accelerator. For the macro-particle simulation, a bunch of particles are generated randomly in three dimentional Gaussian distribution, namely $\left(x, x^{\prime}, y, y^{\prime}, c t, \delta_{p}\right)$. The number of macro-particles in a bunch is tested from 1000 to 1 million, and it is found that the simulation result has enough precision with 10k particles per bunch. Normally 50 different seeds is applied to improve statistical precision. The transverse halos and longitudinal tails of the generated bunch are cut at $4 \sigma$. The normalized transverse emittance is set to be either $5 \mu m$ (current value) or $1 \mu \mathrm{m}$ (expected value after electron photoinjector upgrade), in both horizontal and vertical planes. The RMS energy spread is $1 \times 10^{-3}$ at a low bunch charge of $20 p C$. The RMS bunch length is $100 \mu m$ here, although in the simulation there is no longitudinal motion taken into account.

\subsection{Possible sources for emittance growth}

Four candidates of the emittance growth sources are: first, steering method (one-to-one or one-to-few); second, Chicane Dipole Sextupole field (CDS); third, Quadrupole Dipole Sextupole field (QDS); and fourth, Quadrupole Higher-order fields (QHP). The real emittance growth could be from the combination of these several effects. In the following, the effect of these four individual cases and their combinations is studied by simulation.

It is possible for both dipole and quadrupole magnets to have misalignment. Here a reasonable RMS value of $1 \mathrm{~mm}$ is used, which is based on measurements [3]. For the Quad Higher-order fields (QHP), the strength used in the simulation is $0.8 \% 12^{\text {th }}$ pole and $0.18 \% 20^{\text {th }}$ pole at a radius of $R=1 \mathrm{~cm}$, which is relative to the main quad field [4]. A general formula for higher order fields (with normal and skew components) is listed below.

$$
B_{y}+i B_{x}=B \sum_{n=1}^{10}\left(b_{n}-i a_{n}\right)\left(\frac{x}{r_{0}}+i \frac{y}{r_{0}}\right)^{n-1}
$$

where $B$ denotes the magnetic field on the reference trajectory, $b_{n}$ the normal multipole coefficient, and $a_{n}$ the skew multipole coefficient. The index $n$ is associated with the $2(n+1)$ pole.

The normalized magnet multipole strength is defined as

$$
K_{n}=\frac{\partial^{n} B / \partial x^{n} \cdot L}{B \rho[2 k G-m]}
$$

where $K_{n}$ denotes the normalized magnet multipole strength, $\partial^{n} B / \partial x^{n}$ field gradient, $L$ magnetic length of this element, and $B \rho$ the beam rigidity (roughly $2 k G-m$ for a beam energy of $60 \mathrm{MeV}$ ).

The Chicane Dipole Sextupole field is from the finite pole width of the dipole, as illustrated in Figure 3 (left) which is a real picture of the NLCTA chicane dipole magnet. One could apply an RMS value of $0.085 \%$ Sextupole/Dipole ratio at a radius of $R=1 \mathrm{~cm}$, based on the magnetic higher order fields measurement [4].

Finally, the calculation of the Quad Dipole Sextupole field (QDS) is a bit tricky, as the coils of the dipole corrector are attached on the quadrupoles and share the same iron pole with the quadrupole, as shown in Figure 3 (right). That makes the sextupole field strength of the quadrupole steering magnets a function of the quadrupole strength itself. According to measurement [4], the relation is 1.8\% Sextupole/Quad ratio for a quad current of $200 A$ (integrated field $=57 \mathrm{k} G)$ and a corrector current of $6 A(40 G-m)$, also at a radius of $R=1 \mathrm{~cm}$.

$$
\text { ratio }=1.8 \% \cdot \frac{\theta_{\text {corr }} / 0.02}{B_{\text {quad }} / 57}
$$

where $\theta_{\text {corr }}$ denotes the steering corrector strength in radian, $B_{\text {quad }}$ the quadrupole pole-tip field in kG. 

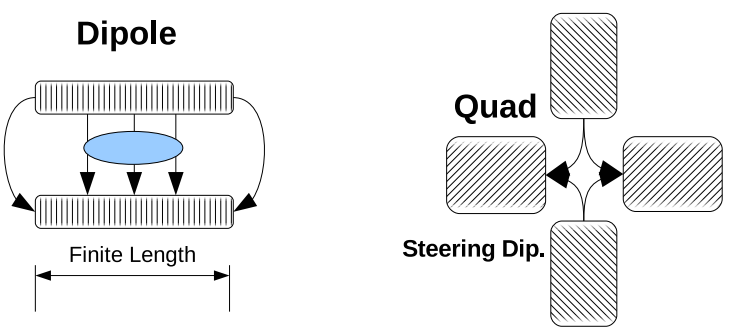

Figure 3: Left: the finite pole width of the chicane dipole; Right: the coils of dipole corrector are attached on the quadrupoles and share the same iron pole with the quadrupole.
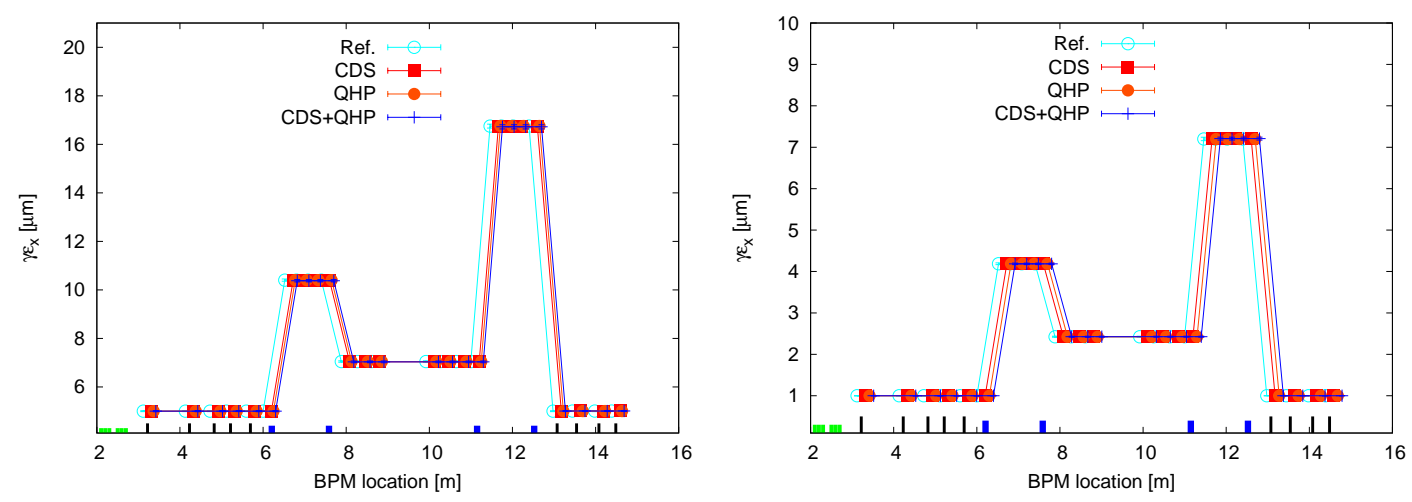

Figure 4: Emittance evolution for four different cases (50 random seeds): ref, reference without any errors; CDS, Chicane Dipole Sextupole field; QHP, Quad Higher-order fields; CDS+QHP. Left: initial normalized emittance is 5 $\mu m$; right: initial normalized emittance is $1 \mu \mathrm{m}$. No emittance grow is observed.

\subsection{Emittance growth without quadrupole offset}

First of all, the emittance dilution is studied without any quadrupole offset. 50 random seeds are used for the higher order fields of dipoles and quadrupoles. In that case, there is no dispersive emittance growth from quadrupoles. Also the Quad Dipole Sextupole field (QDS) is not included as the steering magnets are not used. The emittance evolution is shown in Figure 4 (left), for four different cases (namely ref., CDS, QHP, and CDS+QHP), and with an initial normalized emittance of $5 \mu \mathrm{m}$. Here emittance means normalized projected emittance, if not particularly specified. It is observed that with either CDS or QHP, the emittance evolution is the same with the reference, and only the contribution from the dispersion makes the projected horizontal emittance larger between the dipole magnets. After exiting the chicane, the trajectory-energy correlation is gone and the emittance is recovered to be the initial value. In Figure 4 (right), a similar case is shown with an initial normalized emittance of $1 \mu \mathrm{m}$.

\subsection{Emittance growth with one-to-few steering technique}

In this section, a one-to-few steering technique is applied and then the simulation result on emittance dilution is described. In comparison, the result with one-to-one steering method is reported in the next section. The beam is steered from the center of the first quadrupole to the center of the fifth quadrupole. In the simulation, a one-to-few steering algorithm is written which zero the BPM reading by iteration in calculating the steering corrector's strength. 


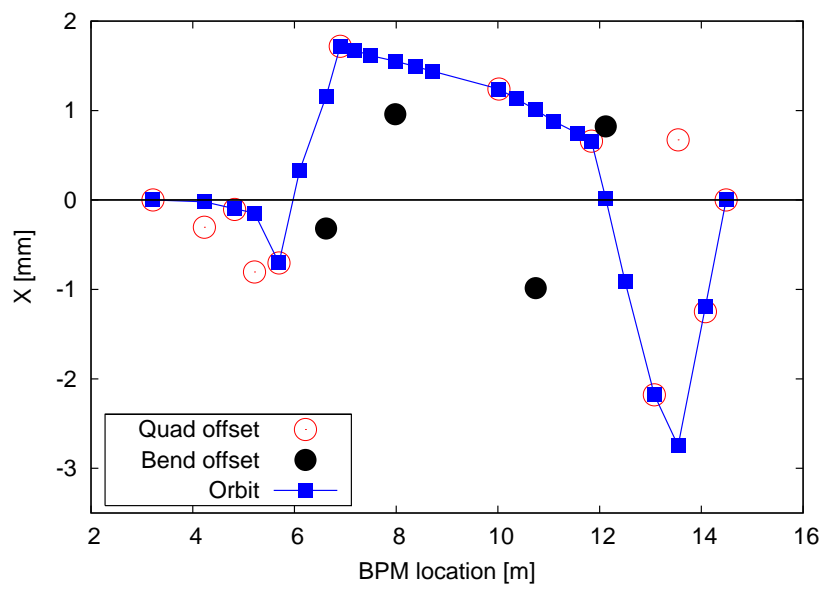

Figure 5: Horizontal beam orbit and the quadrupole (dipole) horizontal offset indicate that the one-to-few steering algorithm works well.
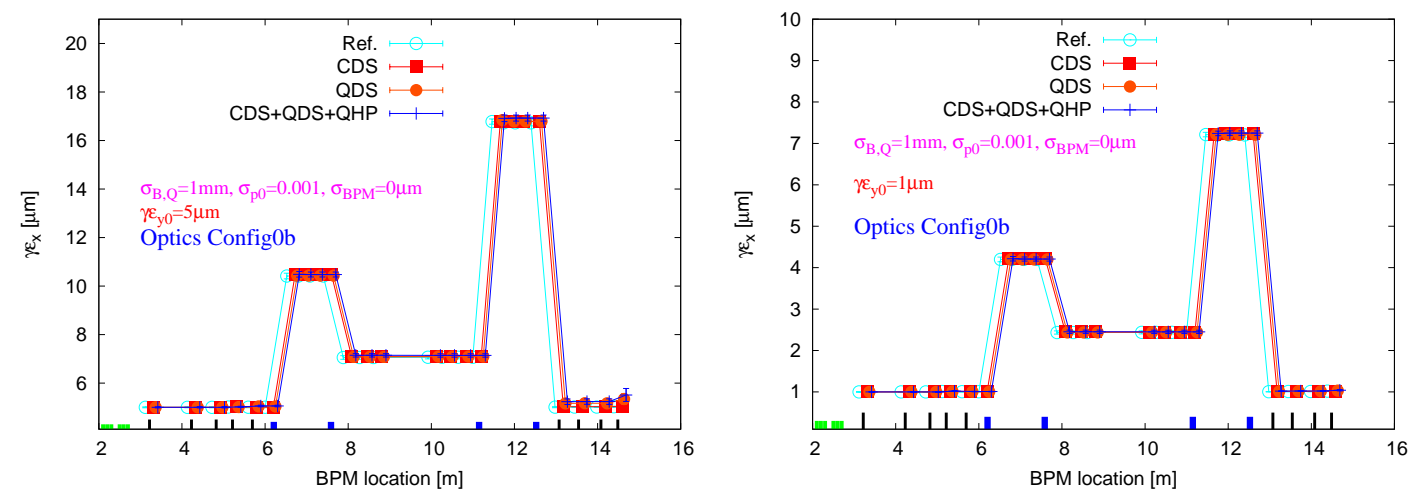

Figure 6: Emittance evolution for four different cases (50 random seeds): ref, reference without any errors; CDS, Chicane Dipole Sextupole field; QDS, Quad Dipole Sextupole field; CDS+QDS+QHP. Left: initial normalized emittance is $5 \mu m$; Right: initial normalized emittance is $1 \mu \mathrm{m}$. One-to-few steering algorithm is applied.

The beam trajectory and quadrupole (dipole) offset are shown in Figure 5.

The beam parameters are the same as described above. Again 50 random seeds are employed for the higher order fields of dipoles and quadrupoles, and also for the quadrupole and dipole offsets. The emittance dilution is shown in Figure 6 , for two cases, with an initial emittance of $5 \mu m$ and $1 \mu m$, respectively.

With one-to-few steering method applied, there is only a small emittance growth, which are $10 \%$ for $\gamma \epsilon_{0}=5 \mu m$, and $4 \%$ for $\gamma \epsilon_{0}=1 \mu \mathrm{m}$. That is due to the fact that the emittance growth is mainly from higher order fields which have a larger impact when the beam size is larger.

The emittance dilution results are also summarized in Table 1 and Table 2, for different cases, and with different initial emittance. One could conclude that the Quad Higher-order fields (QHP) almost adds no emittance growth, and the emittance growth is mainly from sextupole fields (CDS+QDS).

Table I: Emittance growth summary for $\gamma \epsilon_{0}=5 \mu \mathrm{m}$. One-to-few steering method applied.

\begin{tabular}{r|l|l}
\hline Case $\left(\gamma \epsilon_{0}=5 \mu m\right)$ & $\Delta \gamma \epsilon / \gamma \epsilon$ (w/o Q offset) & $\Delta \gamma \epsilon / \gamma \epsilon$ (w Q offset) \\
\hline CDS & $0.1 \%$ & $0.7 \%$ \\
QDS & N $/ \mathrm{A}$ & $6.8 \%$ \\
QHP & $0.07 \%$ & N/A \\
CDS+QDS+QHP & $0.15 \%$ & $10.2 \%$ \\
\hline
\end{tabular}




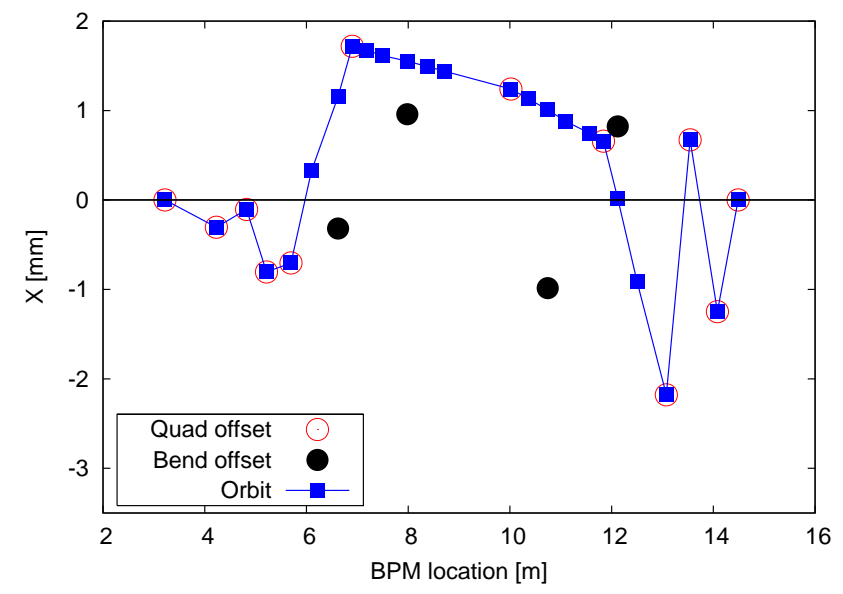

Figure 7: Horizontal beam orbit and the quadrupole (dipole) horizontal offset indicate that the one-to-one steering algorithm works well.

Table II: Emittance growth summary for $\gamma \epsilon_{0}=1 \mu \mathrm{m}$. One-to-few steering method applied.

\begin{tabular}{r|l|l}
\hline Case $\left(\gamma \epsilon_{0}=1 \mu m\right)$ & $\Delta \gamma \epsilon / \gamma \epsilon$ (w/o Q offset) & $\Delta \gamma \epsilon / \gamma \epsilon$ (w Q offset) \\
\hline CDS & $0.08 \%$ & $1.9 \%$ \\
QDS & N $/ \mathrm{A}$ & $3.3 \%$ \\
QHP & $0.06 \%$ & $\mathrm{~N} / \mathrm{A}$ \\
CDS+QDS+QHP & $0.08 \%$ & $4.0 \%$ \\
\hline
\end{tabular}

\subsection{Emittance growth with one-to-one steering technique}

At the starting of each operation period of NLCTA, the electron beam is steered through the linac to get an optimized beam trajectory. Normally one-to-one steering method is applied, which makes use of the steering dipole corrector attached on the quadrupoles. The beam trajectory is then steered from the center of one BPM (attached on one quadrupole) to the center of the next BPM (attached on the next quadrupole). In the simulation, the BPM readings are zeroed by using iteration and a normal iteration time is less than 10 .

In Figure 7, the beam trajectory is shown after one-to-one steering, with all the BPM reading equal zero. For the first five quadrupoles, the beam is steered from the center of one to the next. No BPM resolution nor BPMto-quadrupole offset is considered here. During the commissioning/operation of NLCTA, the BPM-to-quadrupole offset is measured with Beam Based Alignment (BBA) method which measures the BPM response while changing the quadrupole strength. As there is no BPM attached on the chicane dipole magnet, the beam is steered to the center of one quadrupole after/before each dipole magnet, as illustrated in Figure 7. For the last four quadrupoles, again the beam is steered from the center of one to the next.

Similarly, 50 seeds of random errors are taken into account for both higher order fields and magnets offset. With an initial emittance of $\gamma \epsilon_{0}=5 \mu \mathrm{m}$, the emittance dilution in both horizontal and vertical plane is shown in Figure 8. It is observed that the projected emittance [2] equals the linear dispersion corrected emittance [2] (which subtract the linear energy correlation), after the exit of the chicane region. That fact indicates that the emittance growth is mainly from nonlinear effect (higher order fields), which has a similar impact on both horizontal and vertical plane (not only the bending plane).

Including all the higher order fields (CDS+QDS+QHP), and choosing an initial emittance of $\gamma \epsilon_{0}=1 \mu m$, the emittance dilution is shown in Figure 9, again with 50 random seeds. It is observed that the emittance growth is larger with one-to-one steering method than the case with one-to-few steering method.

Lastly, the linear Dispersion Corrected horizontal emittance dilution is shown in Figure 10, to further compare the 


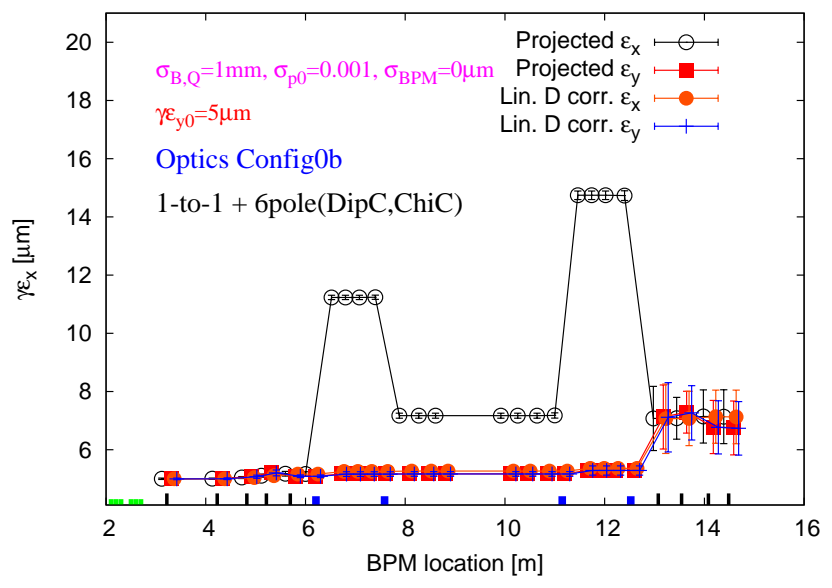

Figure 8: Horizontal and vertical emittance dilution, with sextupole fields (CDS+QDS).

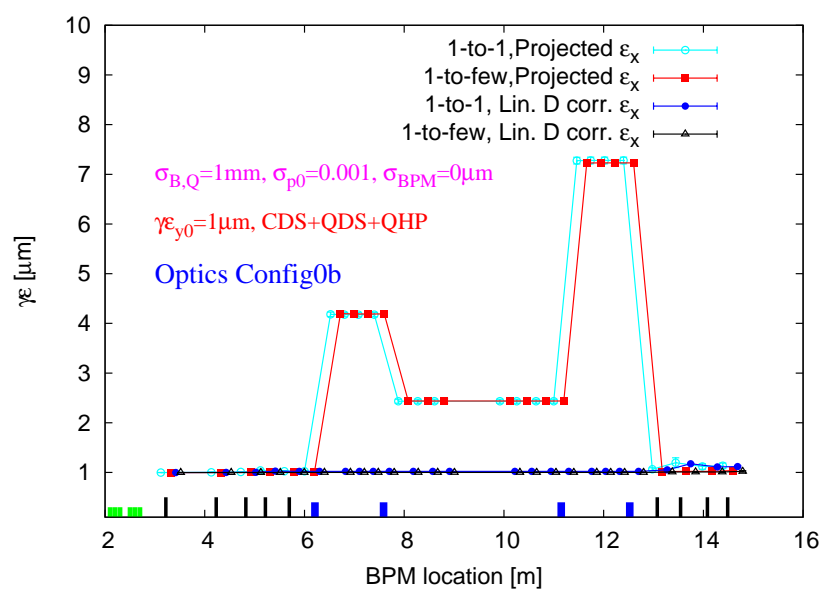

Figure 9: Projected and Linear Dispersion Corrected horizontal emittance dilution, with all the higher order fields $(\mathrm{CDS}+\mathrm{QDS}+\mathrm{QHP})$. With one-to-one or one-to-few steering method.

cases with one-to-one and one-to-few steering techniques. For the current NLCTA running mode which has an initial emittance of $\gamma \epsilon_{0}=5 \mu \mathrm{m}$, the emittance is almost doubled, after passing by the chicane and assuming one-to-one steering method applied.

In order to better preserve the beam emittance, one could apply one-to-few steering method, instead of one-to-one. At the same time, a beam with smaller emittance from the injector has an advantage in further suppressing the higher order fields impact.

\section{Acknowledgement}

The authors would like to thank T. Raubenheimer, M. Woodley, E. Colby, and D. Xiang for helpful discussions. This work was supported by the DOE under Contract DE-AC02-76SF00515.

\section{References}

[1] NLCTA Conceptual Design Report, SLAC-Report-411, 1993.

[2] Yipeng Sun et al., Linac alignment: 1-to-1 correction, SLAC-PUB-14322, 2010. 


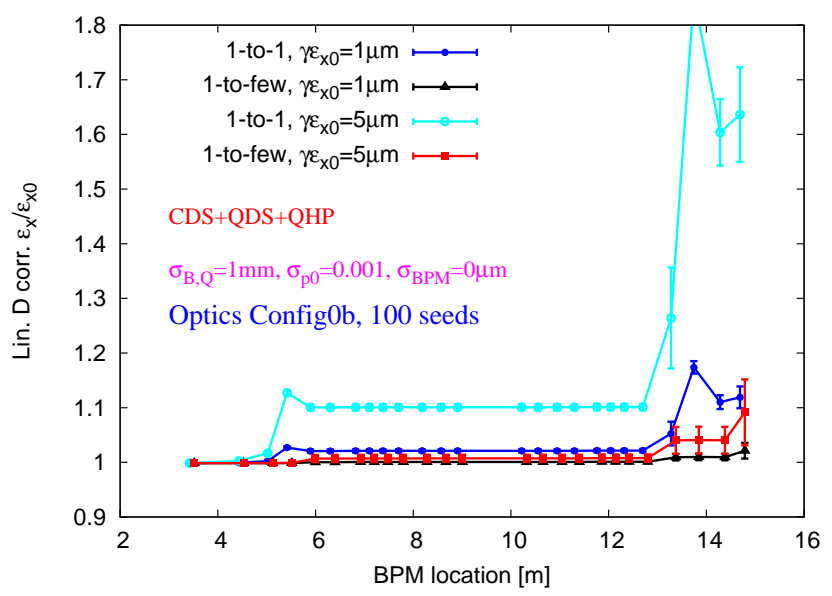

Figure 10: Linear Dispersion Corrected horizontal emittance dilution, with all the higher order fields (CDS+QDS+QHP). With one-to-one or one-to-few steering method. The initial emittance is either $\gamma \epsilon_{0}=5 \mu \mathrm{m}$ or $\gamma \epsilon_{0}=1 \mu \mathrm{m}$.

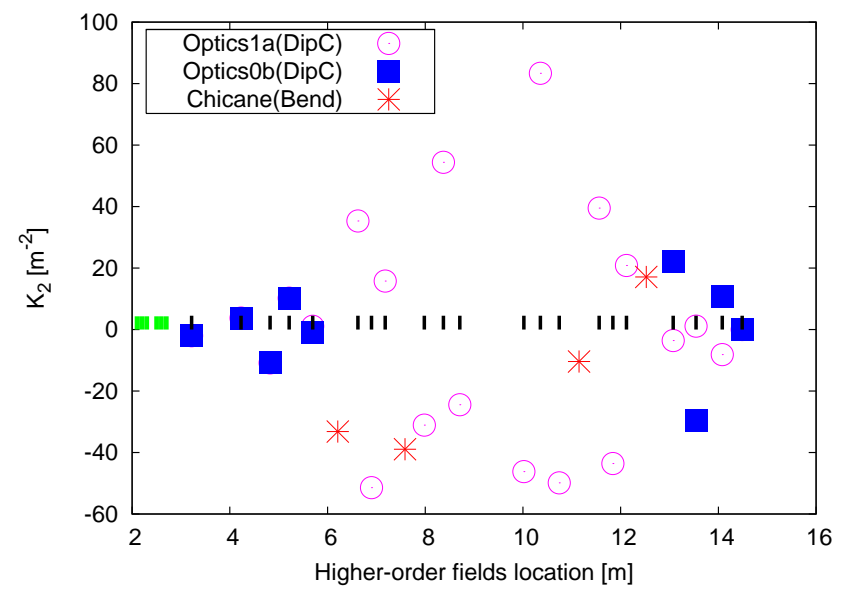

Figure 11: Typical sextupole field strength, for CDS or QDS.

[3] Mark Woodley, private communication, 2010.

[4] Chris Adolphsen et al., NLCTA magnets measurement databook, NLCTA control room, since 1992.

\section{: Appendix ATypical sextupole field strength}

As shown in Figure 11, the normalized integrated sextupole field strength (one specified random seed) is less than $80 m^{-2}$, for both CDS and QDS.

\section{: Appendix BAnalytical estimation}

Based on the analytical formulae which is derived in [2], the emittance dilution is estimated and shown in Figure 12. It is observed that even with a RMS energy spread of $1 \%$ (10 times of the real value), the emittance is only increased by $50 \%-80 \%$. For a RMS energy spread of $0.1 \%$, the emittance growth from quadrupole offset is almost negligible. 


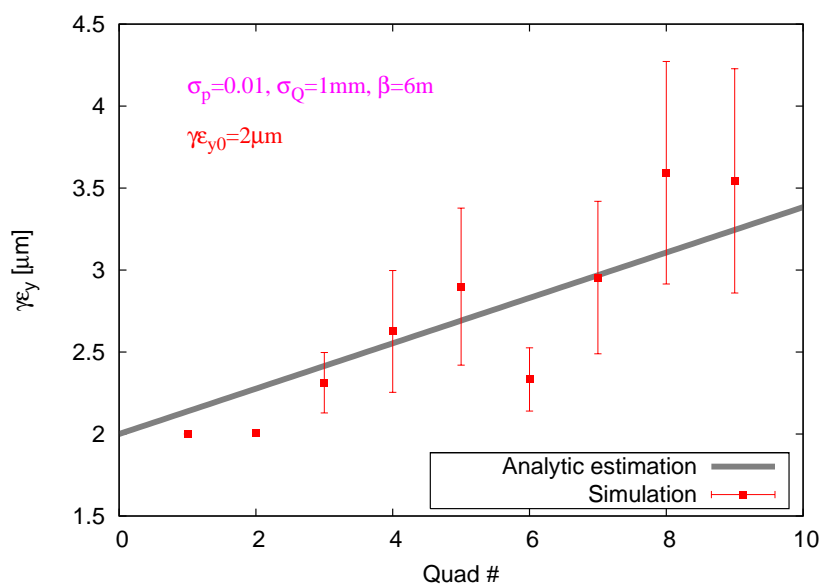

Figure 12: Analytical estimation of emittance dilution. RMS energy spread is assumed to be $1 \%$. 